\title{
Atomic alignment: New Diagnostics of Magnetic Field in Diffuse Medium
}

\author{
Huirong Yan and A. Lazarian \\ ${ }^{1}$ University of Arizona, 1629 E University Blvd, Tucson, 85719, USA \\ email: yan@lpl.arizona.edu \\ ${ }^{2}$ University of Wisconsin-Madison, 475 Charter St., Madison, 53706, USA \\ email: alazarian@wisc.edu
}

\begin{abstract}
We discuss a new technique of studying magnetic fields in diffuse astrophysical media, e.g. interstellar and intergalactic gas/plasma. This technique is based on the angular momentum alignment of atoms and ions in their ground or metastable states. The alignment reveals itself in terms of the polarization of the absorbed and emitted light. The corresponding studies of magnetic fields can be performed with multiband spectropolarimetry, from UV, optical, to IR/radio. A unique feature of these studies is that they can reveal the 3D orientation of magnetic field. We mention several cases of interplanetary, circumstellar and interstellar magnetic fields for which the studies of magnetic fields using ground state atomic alignment effect are promising.
\end{abstract}

Keywords. ISM: magnetic fields, polarization, atomic processes.

\section{Conditions for atomic alignment}

The basic idea of the atomic alignment is quite simple. The alignment is caused by the anisotropic deposition of angular momentum from photons. In typical astrophysical situations the radiation flux is anisotropic. As the photon spin is along the direction of its propagation, we expect that atoms scattering the radiation from a light beam to be aligned (see Hawkins 1955; Varshalovich 1968). Such an alignment happens in terms of the projections of angular momentum to the direction of the incoming light. For atoms to be aligned, their ground state should have non-zero angular momentum. Therefore fine (or hyperfine) structure is necessary to enable various projection of atomic angular momentum to exist in their ground state.

Owing to the magnetic precession, the atoms with different projections of angular momentum will be mixed up. Magnetic mixing happens if the angular momentum precession rate $\nu_{L}$ is higher than the rate of the excitation from the ground state $\tau_{R}^{-1}$, which is true for many astrophysical conditions, e.g., interplanetary medium, ISM, intergalactic medium, etc. As the result, angular momentum is redistributed among the atoms, and the alignment is altered according to the angle between the magnetic field and radiation field $\theta_{r}$.

Long-lived alignable metastable states that are present for some atomic species between upper and lower states may act as proxies of ground states. Absorptions from these metastable levels can also be used as diagnostics for magnetic field therefore.

\section{Range of applicability}

Many species can be aligned. The corresponding lines (including both absorption and emission) can be used as the diagnostics. A number of lines with the maximum degree of polarization have been provided in YLa,b,c, Yan \& Lazarian 2008b. An incomplete list of objects where effects of alignment should be accounted for arises from our studies (YLa,b,c). These include diffuse medium in the early Universe, quasars, AGNs, reflection nebulae, high and low density ISM, circumstellar regions, accretion disks and comets. One can easily add more astrophysical objects to this list. For instance, Io sodium tail can be studied the same way as sodium tail of comets (YLb).

In general, in all environment when optical pumping is fast compared with the collisional processes we expect to see effects of atomic alignment and magnetic realignment of atoms. The 
a)

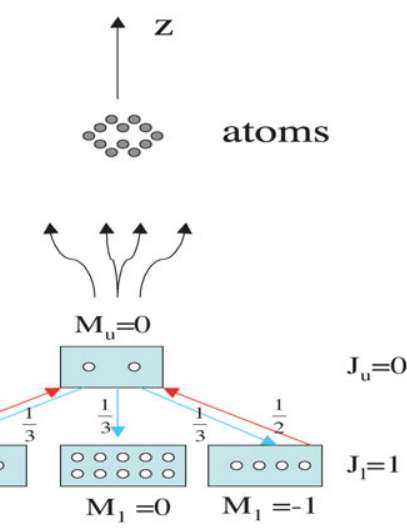

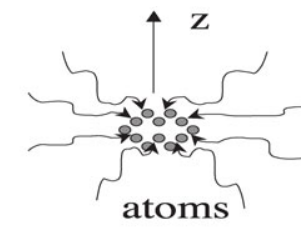

Pumping source

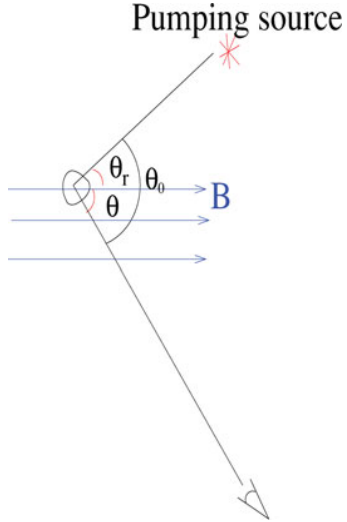

Figure 1. Left: A toy model to illustrate how atoms are aligned by anisotropic light. Atoms accumulate in the ground sublevel $M=0$ as radiation removes atoms from the ground states $M=1$ and $M=-1$; right: Typical astrophysical environment where the ground-state atomic alignment can happen. A pumping source deposits angular momentum to atoms in the direction of radiation and causes differential occupations on their ground states. In a magnetized medium where the Larmor precession rate $\nu_{L}$ is larger than the photon arrival rate $\tau_{R}^{-1}$, however, atoms are realigned with respect to magnetic field. Atomic alignment is then determined by $\theta_{r}$, the angle between the magnetic field and the pumping source. The polarization of scattered line also depends on the direction of line of sight, $\theta$ and $\theta_{0}$. (From YLc)

wide variety of atoms with fine and hyperfine structure of levels ensures multiple ways that the information can be obtained. Comparing information obtained through different species one can get deep insights into the physics of different astrophysical objects. If the implications of atomic alignment influenced the understanding of particular features of the Solar spectrum (Landi Degl'Innocenti E. 1999, Trujillo Bueno 1999), then the studies of atomic alignment in a diffuse astrophysical media can provide much deeper and yet unforeseen changes in our understanding of a wide variety of physical processes.

As the resolution and sensitivity of telescopes increases, atomic alignment will be capable to probe the finer structure of astrophysical magnetic fields including those in the halo of accretion disks, stellar winds etc. Space-based polarimetry should provide a wide variety of species to study magnetic fields with.

\section{References}

Hawkins, W. B. 1955, Phys. Rev. 98, 478

Landi Degl'Innocenti E. 1999, in Solar Polarization, K. N. Nagendra, \& J. O. Stenflo, eds. (Kluwer Academic Publisher), p. 61

Trujillo Bueno, J. 1999, in Solar Polarization, K. N. Nagendra, \& J. O. Stenflo, eds. (Kluwer Academic Publisher), p. 73

Varshalovich, D. A. 1968, Astrofizika, 4, 519

Yan, H. \& Lazarian, A. 2006, ApJ, 653, 1292 (YLa)

Yan, H. \& Lazarian, A. 2007, ApJ, 657, 618 (YLb)

Yan, H. \& Lazarian, A. 2008a, ApJ, 677, 1401 (YLc)

Yan, H. \& Lazarian, A. 2008b, in Magnetic Fields In The Universe II, RevMexAA, astro$\mathrm{ph} / 0806.3703$ (YLd) 